\title{
Makers experiences: Upcycling, interfaces and reactive devices in Industrial Design
}

\section{SIGRADI2018 TECHNOPOLITICAS \\ xxii congresso da sociedade iberoamericana de gráfica digital 22th conference of the iberoamerican society of digital graphics 07|08|09|novembro|2018 iau usp | são carlos | sp br}

\author{
Mauro Chiarella \\ CONICET-Universidad Nacional del Litoral| Argentina | mchiarella@hotmail.com \\ Sebastian Martini \\ Universidad Nacional del Litoral| Argentina | sebamartinidis@gmail.com \\ Matías Dalla Costa \\ Universidad Nacional del Litoral| Argentina | matiasdallacosta@hotmail.com \\ Martín Veizaga \\ Universidad Nacional del Litoral| Argentina | veizagamartin@hotmail.com
}

\begin{abstract}
The Maker culture emphasizes collaborative learning and distributed knowledge. Expands exponentially the multiplicity of resources and possible processes. Finding effective and efficient ways to use them to develop predictive models that focus decision-making towards performance-oriented designs is the new challenge. Through the application of didactic strategies of problem-based learning will analyze five experiences of the degree of Industrial Design, Course IMD, National University of the Littoral based on logic of personalized manufacturing, augmented graphic thinking and collective creation.
\end{abstract}

Keywords: Crowthinking; Upcycling; Arduino; Visual programming

\section{INTRODUCCIÓN}

La creciente importancia del análisis de datos en la sociedad de la información es uno de los paradigmas de nuestra época. Encontrar maneras efectivas y eficientes de analizarlos para desarrollar modelos predictivos que focalicen la toma de decisiones hacia diseños orientados al rendimiento, son propuestos nuevamente como enfoques integradores e innovadores para la resolución de problemas proyectuales. La optimización de medios y recursos nos conduce a trabajar indirectamente con una realidad mediada a través de sus registros y procesos. Históricamente necesitábamos más datos de lo que teníamos. Hoy, por primera vez, parece que tenemos más datos de lo que necesitamos. Como administrarlos, procesarlos y utilizarlos estratégicamente sigue siendo el desafío.

Podemos calcular, fabricar y componer variaciones personalizadas en todas las escalas con materiales industriales que fueron estandarizados para la producción en masa, inclusive simplificando y automatizando la ejecución de complejas geometrías no seriadas. El computacional making nos permite crear nuestros propios instrumentos proyectuales mediante la sustitución; adaptación y generación de dispositivos; prótesis robóticas y máquinas de control numérico. Es así como el pensamiento gráfico aumentado aparece integrando procesos algorítmicos, heurísticos y de manufactura. Amplía horizontes mediante los recursos instrumentales de la representación, la simulación, el diseño computacional, la manufactura digital y el computational making. (Chiarella, 2018)

La cultura Maker enfatiza el aprendizaje colaborativo y el conocimiento distribuido. Posibilita la capacidad de ser pequeño y al mismo tiempo mundial; artesanal e innovador; de alta tecnología y de bajo coste. Los intereses comunes incluyen desarrollos en electrónica, robótica, impresión $3 \mathrm{D}$ y el empleo de máquinas $\mathrm{CNC}$, como así también el uso de herramientas clásicas como el trabajo de herrería, tornería, carpintería y las tradicionales artes y oficios. Su interacción con la comunidad y el intercambio de conocimientos se haya mediado por las tecnologías de trabajo en red. Los sitios web y las redes sociales son la base del repositorio de conocimientos y el canal central del intercambio de ideas e información. Como sabemos, la nube en sí es una herramienta al servicio del movimiento creador, lo que permite una mayor colaboración, flujo de trabajo digital, y la manufactura compartida mediante la descarga y distribución de archivos. Bajo los conceptos enunciados se analizarán experiencias y ejercicios realizados en la carrera de Diseño Industrial, cátedra $x x x$, de la Universidad xxx basadas en lógicas de fabricación personalizada, pensamiento gráfico aumentado, aprendizaje colaborativo, conocimiento distribuido y creación colectiva.

\section{MAKERS Y EDUCACION}

La cultura Maker, en el campo educativo, se ve reflejada en un sinfín de experiencias y espacios en los cuales se estimula la indagación junto a la tecnología. Dale Dougherty (2013) en su artículo "The Maker Mindset" la denomina "Experimental Play" (juego experimental) a raíz de la experiencia de observar como los Makers "jugaban" con la tecnología para aprender sobre ello. Se toma a una nueva tecnología como una invitación a jugar en el cual encontramos una satisfacción al hacerlo. Los Makers exploran, intervienen y crean junto a la tecnología, 
inclusive funciones y operaciones que no estaban previstas originalmente.

Sus raíces teóricas se remontan a las Escuela Nueva o Escuela Activa. Impulsada por la revolución francesa, las teorías de Darwin y la planteamientos de la psicología de la Gestalt. Así es como diferentes autores, que indagan entorno a la cultura Maker y la educación, como Blikstein (2013), P; Sheridan, K (2014); Martin, L (2015), identifican a Dewey, J; Frobel, F; Montessori, M; Piaget, J; entre otros, como los primeros referentes de modelos educativos centrados en los estudiantes, en los cuales el sujeto deja de ser objeto de la práctica educativa para convertirse en sujeto de desarrollo de sí mismo (Villamil, 2008:6). Lo que se propone, dentro de éstos métodos educativos activos, es el hacer como condición para el aprendizaje. La práctica implica la manipulación de conocimientos para lograr un objetivo propuesto por el sujeto en donde el docente asume el rol de guía y facilitador de instrumentos para que los sujetos construyan sus propias realidades.

Los Makers investigan y aprenden haciendo. Riviere Ángel (1984: 49-54) define en palabras de Vygotski en su "psicología del Juego", el juego como instrumento y recurso socio-cultural, se consolida como un elemento promotor del desarrollo mental, facilitando el desarrollo de operaciones superiores de entendimiento y ampliando lo que denomina Zona de Desarrollo Próximo.

En la teoría constructivista de Jean Piaget y, más directamente, en el construccionismo de Seymour Papert, podemos hallar los referentes teóricos directos de los modelos de enseñanza Maker. Sobre la base teórica del constructivismo de Piaget, la cual afirma que el conocimiento no resulta del acto de recibir información sino de un proceso de internalización mediante la creación de significados productos de la exploración, el construccionismo de Papert, afirma que el aprendizaje se relaciona de forma intrínseca con la producción de artefactos que funcionan como elementos activadores del proceso de construcción del conocimiento. El construccionismo de Papert comparte con el constructivismo la idea que el aprendizaje es el resultado de la construcción de estructuras de conocimiento a través de la progresiva interiorización de acciones (Tesconi, S 2015:5).

Los Maker buscan oportunidades para aprender nuevos conocimientos mientras hacen con sus propias manos. Son proactivos, creativos e inclusivos en espíritu. El impulso nace desde el interés personal pero creen que a través del trabajo colaborativo y compartiendo en comunidad es cuando pueden lograr todo lo que imaginan. El hacer se constituye como elemento motivador, el sujeto elije sus propios retos y se responsabiliza de su aprendizaje para resolver nuevos problemas.

\section{METODOLOGIA}

La cátedra IMD-DI en continuidad con los Fundamentos de Cátedra, argumentaciones e investigaciones pioneras sobre los MD y TIC's, iniciadas en IMD-FADU-UNL (Stipech, 2012), ha desarrollado a lo largo de sus primeros 5 años, un trabajo práctico por cuatrimestre. Estos se han basado sobre problemáticas conceptuales e instrumentales derivadas de la incorporación estratégica de las nuevas tecnologías a los procesos proyectuales en la carrera de Diseño Industrial. Los mismos se desarrollan en 3 etapas: a) Investigación y estudio de la problemática, b) Experimentación de Proceso de aproximación proyectual propuesto por la cátedra, c) Prueba de lógicas de prototipado sugeridas.

Durante el desarrollo de los ejercicios se explora, además, sobre lógicas implícitas en la cultura Maker articulando las prácticas propuestas por la asignatura, con potencialidades que permiten enriquecer transformaciones y desarrollos didácticos. Cada trabajo práctico, en sus contenidos, enfocan la problemática, no solo en el aprendizaje de los conocimientos y habilidades necesarias para manipular los múltiples instrumentos proyectuales, sino en la comprensión de lo que estas herramientas de prefiguración suponen para la concepción e ideación del diseño.

\section{EJERCICIO A: INTERFASES CORPOREAS (SECCIONES APROPIABLES)}

Artificios, complementos y prótesis físicas que, a modo de interfase tecnológica, potencian-amplían-alteran las relaciones del cuerpo y su ambiente cercano.
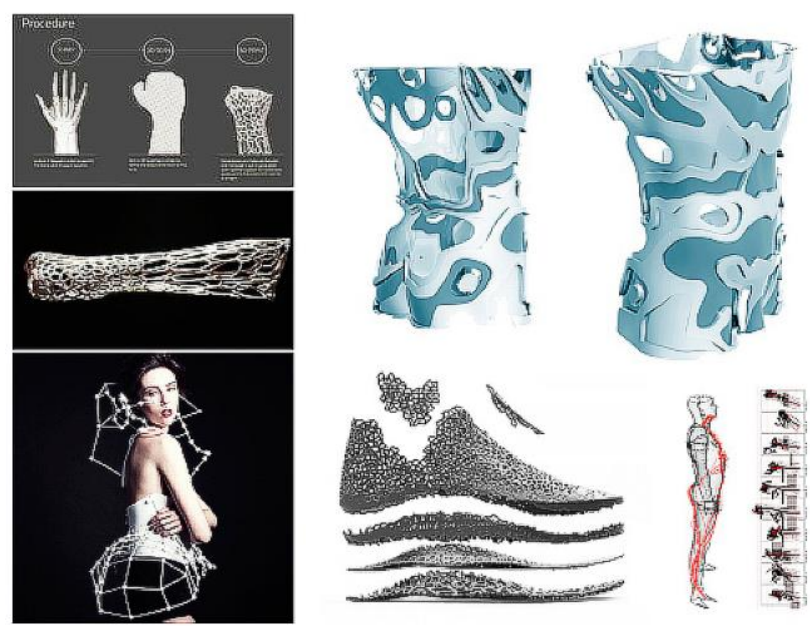

Programación Visual - Ingeniería Inversa - Prótesis

Figura 1: Jake Evil; Francis Bitonti; Marc Fornes \& Theverymany; Earl Stewart; Skingraph. Fuente: Autores.

Problemática: El cuerpo como interfase: "Mi cuerpo, así como el suyo, es un cuerpo tecnológico. A través de él interactúo con los otros, me adapto al ambiente y soy capaz de alterarlo. Además de tecnológico el cuerpo es una interfaz. A lo largo de la historia el hombre elaboró formas de comunicación y lenguajes corporales sofisticados, algunos de las cuales tan eficientes que perduran hasta hoy. A diferencia de otras especies, también inventó una serie de artificios y artefactos capaces de ampliar y transformar la cognición humana y el ambiente: algunos de ellos físicos, otros mentales. Incluyendo el advenimiento del computador, ninguno de ellos existiría sin la capacidad de invención del hombre." (Kutschat Hans).

Proceso de Aproximación Proyectual: NURBS + Diseño Paramétrico. 
Lógicas de prototipado o presentación sugeridas: Presentación Oral + Panel Gráfico

\section{EJERCICIO B: UPCYCLING (REUTILIZACION CREATIVA)}

Transformar objetos sin uso en objetos de calidad mediante el diseño como valor agregado.

Fabricación Personalizada - Conocimiento Distruibuído Emprendimiento
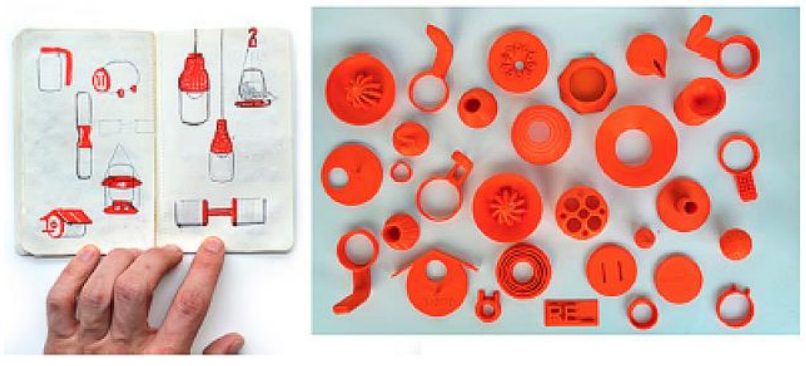

Figura 2: Proyecto RE_(http://project-re.blogspot.nl/) Samuel Nelson Bernier. Fuente: Autores.

Problemática: Customización en masa y Fabricación personal: La customización en masa supone un nuevo paradigma en oposición tanto a la producción artesanal como a la producción en masa que nos ha traído la revolución industrial. La producción en masa ha permitido la producción de grandes cantidades de productos y servicios a bajo coste, mientras que (actualmente) la producción artesanal se reduce a un número limitado de productos o servicios muy individualizados, pero también de mayores costes y plazos de entrega. Requiere una forma completamente diferente de entender y organizar el negocio, y no afecta únicamente al ámbito de producción. Tanto el producto o servicio como el modelo del negocio debe rediseñarse para crear variedad y customización de manera flexible y rápida, es decir, para crear economía de gama, en vez de economía de escala. Producir lo Complejo y lo Singular en forma Simple a partir de la Automatización y Programación de procesos. La Fabricación Personal y los Fab-Labs contribuyen a la customización en masa posibilitando que los diseñadores participen en el propio proceso de manufactura, mediante un modelo de negocio de co-fabricación (DiseñadoresFabricantes-Emprendedores).

Proceso de Aproximación Proyectual: NURBS + Diseño Paramétrico.

Lógicas de prototipado o presentación sugeridas: 3D Printer-Maquina CNC

\section{EJERCICIO C: MICRO-EXPERIENCIAS: DEGUSTACION CREATIVA (UNFOLDING PROCESS)}

Producir lo complejo y lo singular en forma simple a partir de la automatización y programación de procesos

Pensamiento Gráfico Aumentado - Prototipado Rápido Interfases

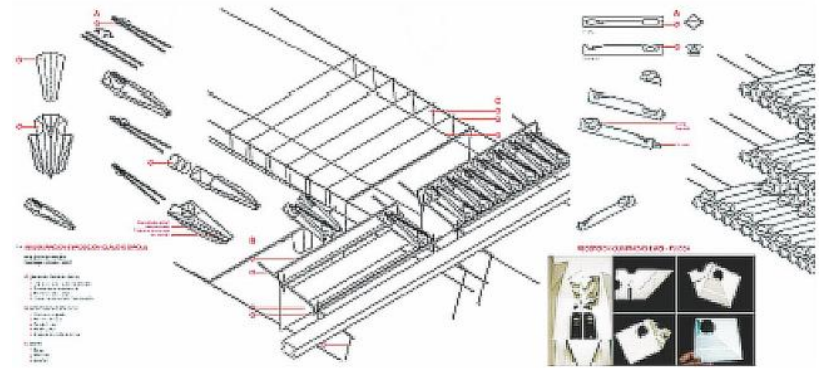

Figura 3: Ricardo Lang Viacava - Diseño, Acto y Celebración. e(ad)-PUCV. Fuente: Autores.

Problemática: Fabricación personalizada de bajo costo. Diseño y Degustación. Cocina, Mesa y Comida: "Sin diseño, alimentarse sería más difícil y aburrido. La alimentación sería mera supervivencia. Respirar y comer son las necesidades imperantes de la vida. Pero sin la ayuda de la creatividad material estaríamos condenados a recoger lo que brota de los árboles y llevárnoslo directamente a la boca. El ingenio humano, el diseño, mejora desde hace siglos y de forma creciente el proceso de la alimentación humana. Abarca todos los ámbitos y todas sus fases. Comenzó contribuyendo a la obtención de los diversos alimentos de cultivo, para los que ha ideado, desde hace milenios, ingeniosos artilugios que hagan más llevaderas estas tareas. También ha generado armas y útiles para la caza y la pesca. Más tarde, el diseño volvió a ser crucial al generar instrumentos para la elaboración y cocción de los alimentos, su manipulado, transformación y conservación. Sin ollas, pucheros o espumaderas, sería extremadamente difícil cocinar. Además, un buen diseño es fundamental para facilitar los elementos de servicio y degustación en la mesa, ya que proporciona los recipientes y contenedores en los que presentar el alimento y llevarlo a la boca. En el espacio de la mesa, el diseño de instrumentos, además de funcional, adquiere un alto grado de simbolismo y representatividad, lo que lo ha abocado a una interesantísima interrelación con el arte $y$ ha dado origen a piezas singulares que las artes decorativas han desarrollado a lo largo de los siglos. El ritual de la mesa" (Juli Capella).

\section{Proceso de Aproximación Proyectual: Unfolding Process}

Lógicas de prototipado o presentación sugeridas: Cortadora Láser + Preparación de la Micro-Experiencia

\section{EJERCICIO D: DISPOSITIVOS REACTIVOS (MICROCONTROLADORES Y SENSORES)}

Un Dispositivo Reactivo está vinculado a la activación por estímulos. Posee una gama de comportamientos predefinidos que se pueden activar produciendo un cambio en el entorno inmediato.

Aprendizaje Colaborativo - Arduino - Prototipado Rápido
3 


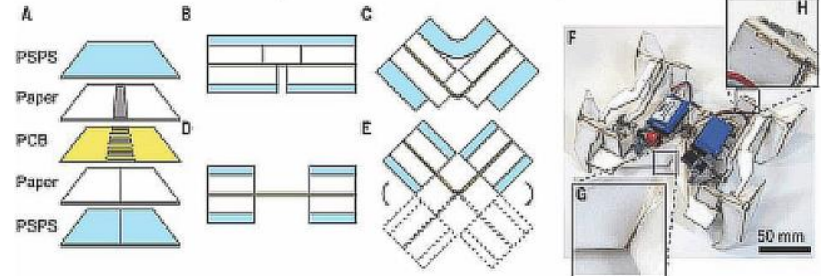

Figura 4: Self-folding robots - Harvard University (http://hvrd.me/A2mM9)

Problemática: Podemos definir dispositivo al aparato, mecanismo o artificio que produce determinadas acciones previstas. Dispositivo digital es un conjunto tecnológico que usa distintas unidades de un sistema de procesamiento de información. El término entrada y salida de información también refiere a la ejecución de acciones u operaciones a través de dichos mecanismos. Un dispositivo de entrada o salida puede ser cualquier tipo de unidad funcional o subsistema que forma parte de un conjunto integral, pudiendo enviar señales o procesar información para establecer distintos tipos de comunicación o reacción interna y externa. Un dispositivo reactivo está vinculado a la activación por estímulos. Los sistemas reactivos son elementos de nuestro entorno que tienen (en diversos niveles de complejidad) una gama de contenidos predefinidos, como comportamientos que se pueden activar para producir un cambio. Los dispositivos reactivos pertenecen a la primera instancia de aprendizaje hacia los dispositivos responsivos 0 inteligentes. El diseño de interacción o responsivo hoy es una evolución de los modelos de diseño reactivo enraizada en los primeros métodos de instrucción en computación; un modelo rígido, lineal y con variables restrictivas.

Proceso de Aproximación Proyectual: Programación de Arduino y sensores.

Lógicas de prototipado o presentación sugeridas: Cortadora Laser + electrónica

\section{EJERCICIO E: SISTEMAS MODULARES VERSATILES (SOPORTE ESTRUCTURAL DINAMICO)}

Exploración de acople modular de mínimos componentes, posibilitante de múltiples iniciativas de diseño y adaptable a diversas condiciones y configuraciones contextuales.

Programación Visual - Fabricación Personalizada Creación Colectiva

Problemática: Programación Visual - Creación Colectiva:

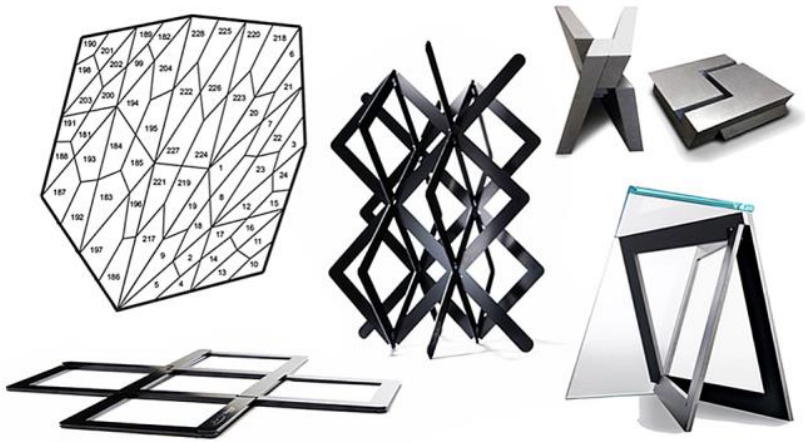

Figura 5: Quadror: A structural support system + Unfolding Process. Fuente: Autores.
En la cultura maker la interacción en la comunidad y el intercambio de conocimientos se encuentra mediado por las tecnologías de trabajo en red. Los sitios web y las redes sociales son la base del repositorio de conocimientos y el canal central del intercambio de ideas e información, hibridado por encuentros físicos en espacios de intercambio social como los Hackerspaces y los Fab Lab. El aprendizaje colaborativo y la cognición distribuida cuestionan el mito de la originalidad exclusiva de autor y su obra. No es necesario crear algo desde cero o tener una idea única y original. Es posible participar en la mejora colectiva o adaptación contextual de ideas o diseños existentes. Los proyectos compartidos en línea inspiran a otros y son una oportunidad de colaboración infinita.

Proceso de Aproximación Proyectual: Diseño Paramétrico

Lógicas de prototipado o presentación sugeridas: Cortadora Laser + mecanismos de articulación y plegado

\section{RESULTADOS}

El desarrollo de las diferentes experiencias delimitadas en ejercicios proyectuales nos permite identificar aquellos puntos de acuerdo y de tensión sobre los aportes de la cultura Maker en entornos formales de aprendizaje.

En las experiencias de aprendizaje basado en problema, los estudiantes aprenden a resolver situaciones reales sobre los que deben tomar decisiones y reflexionar. Establecen hipótesis y proponen ideas, hacen conexiones entre los objetivos y los conceptos para hallar soluciones a los desafíos y los procesos planteados, retienen el conocimiento adquirido, desarrollando sus habilidades.

Siguiendo las categorías enunciadas por Martin (2015), nos referimos a un conjunto de cuatro atributos característicos del Making educativo. Estos cuatro elementos son: Aspecto lúdico, exploración activa, el fallo como instancia de aprendizaje y la experiencia colaborativa.

Aspecto Lúdico: Las experiencias makers nos animan a experimentar jugando promoviendo el uso de instrumentos de código abierto y la fabricación digital conjuntamente al uso de herramientas tradicionales para desarrollar competencias avanzadas en una nueva dinámica de relación con el conocimiento. Los temas cotidianos se transforman el juegos posibles para especular sobre posibles innovaciones de lo diario. Hechos que posibilitan y se potencian con prácticas transdisciplinares, invitan a indagar para descubrir intereses sin temor al fracaso, adoptando al mismo como un medio para el éxito. Según González Böhme y Calvo Barentin (2014) el desarrollo de competencias avanzadas en computación se ha vuelto un objetivo global en la formación de profesionales productivos y de calidad en esta nueva era. Este enfoque es consecuente a las necesidades formativas de diseñadores industriales que se ven enfrentado a cambios científicos-tecnológicos, sociales y económicos constantes. Según Josep Tresserras (2015), la transdisciplinaridad en diseño influye en la formación de diseñadores industriales, el diseño, como consecuencia de su complejidad, incorpora una alta interdisciplinariedad y una fuerte interrelación, especialmente debido a su carácter heurístico e iterativo. 


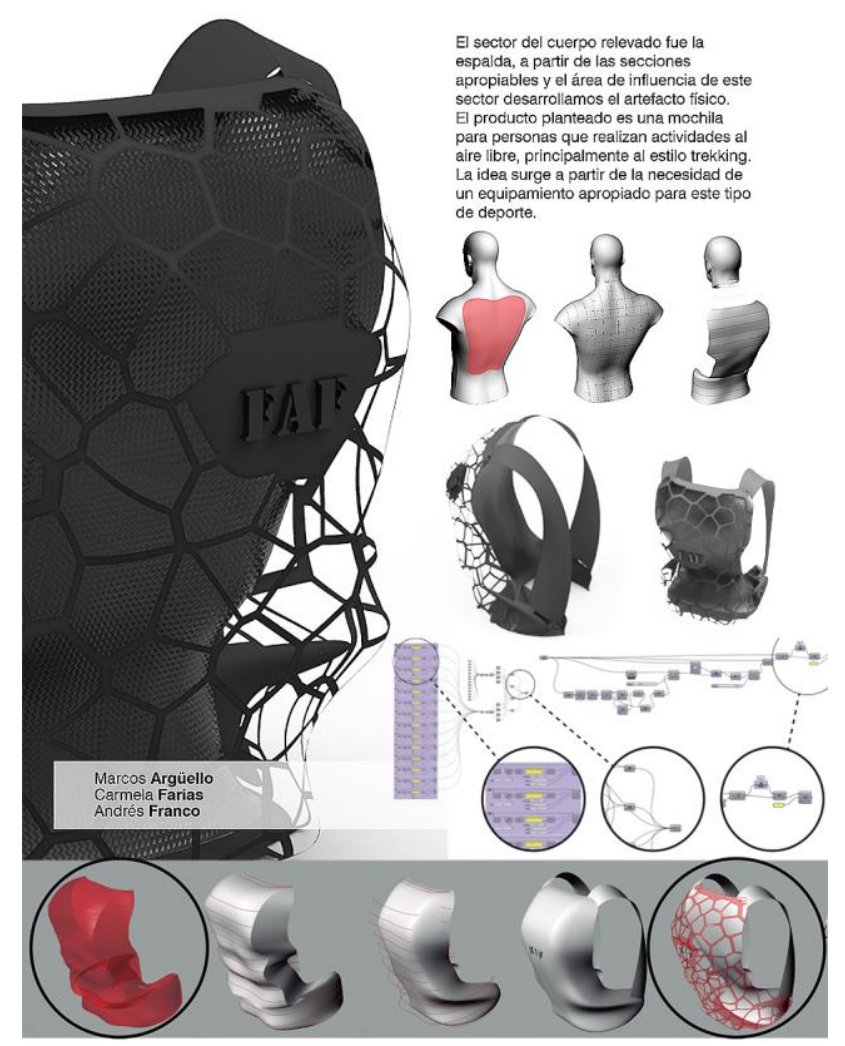

Figura 6: Interfases Corpóreas (Secciones Apropiables). Grupo: Arguello-Farías-Franco. Fuente: Autores.

Exploración Activa: La creatividad vincula recursos humanos y tecnología y depende de la selección y combinación de los elementos que constituyen la materia prima para la innovación. Una nueva forma de aprender y de enseñar deriva potencialmente de las innovaciones tecnológicas. Ya no se establece una base importante de conocimiento en el individuo, en una memoria privada que uno debe llenarla con cosas que va usar en el futuro. Como hoy la memoria es pública (bases de datos, bibliotecas de archivos, web semántica) la información se encuentra disponible y asequible para todos, entonces lo que hay que enseñar es como llegar a ella, como usarla, como reciclarla, acompañando procesos.

Si bien es el estudiante el que debe intervenir en la adquisición de nuevas competencias en una dinámica de trabajo colaborativo y activo frente a una problemática presentada, nuestro rol de acompañar procesos es clave. La técnica del Jigsaw (rompecabezas) ideado por el profesor Elliot Aronson a principios de los '70, aparece como un recurso didáctico posible que nos brinda la secuencia de pasos metodológicos para diseñar nuestros ejercicios. El Rompecabezas es una técnica de aprendizaje cooperativo basada en la investigación. La secuencia de pasos da estructura a la actividad y genera una interdependencia entre los estudiantes. Es esta interdependencia "requerida" entre los estudiantes lo que hace a este método de aprendizaje único. Es la interdependencia la que anima a los estudiantes a tomar parte activa de su aprendizaje al convertirse a ellos mismos en expertos, convirtiéndose en activos valiosos para el resto del grupo.
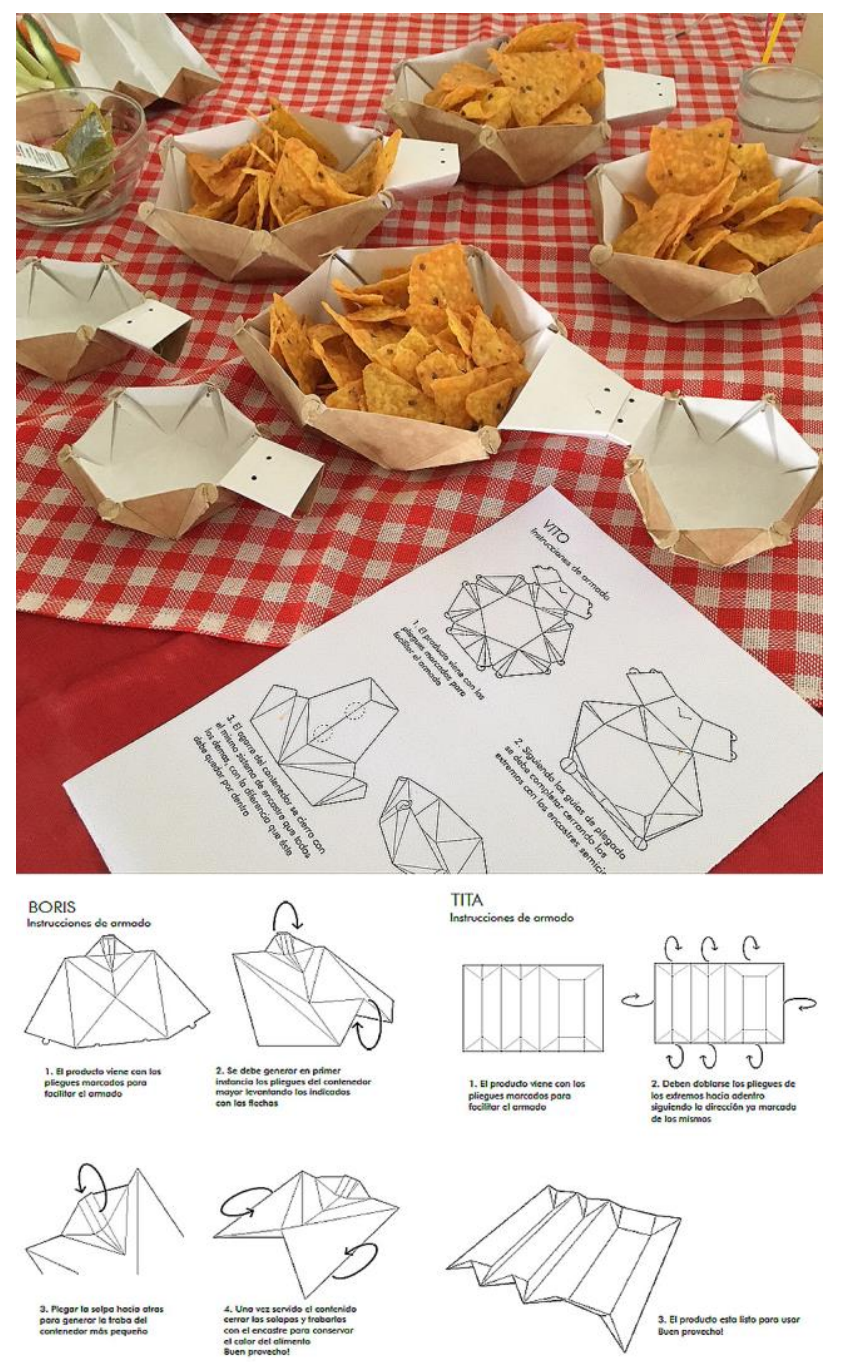

Figura 7: Micro-Experiencias: Degustación Creativa (Unfolding Process). Grupo: Gaviola-Horler-Wingeyer. Fuente: Autores.

Fallo como crecimiento: Esta es la instancia mas compleja de asimilar en el sistema universitario. La universidad, con su sistema riguroso de evaluación, saber compartimentado y secuencialidad controlada de cursado (materias correlativas), no propicia el equívoco como parte de un proceso. A pesar de ello, hay instancias de ida y vueltas dentro del proceso personal del estudiante, que pueden acompañarse en un período controlado mediante instancias de pre-entrega previamente acordadas. La masividad del cursado es el otro gran obstáculo para los seguimientos de procesos personalizados.

Si el texto es un tejido de citas extraídas de innumerables centros de cultura, en el ambiente de producción controlado por software, estas citas ya no vienen solo de los recuerdos del creador, de su memoria visual, auditiva o táctil, sino también directamente de las bases de datos de objetos mediáticos que han sido sociabilizados por el trabajo colectivo en red. Existes valores colectivos en forma de inteligencia común posibilitados por la tecnología. El proceso de diseño en los nuevos medios implica trabajar con diferentes menús de varios programas, bases de datos de objetos digitales, bibliotecas de archivos y fundamentalmente conexión en red. La sociabilización y reciclaje de procesos erráticos 
podría compensar la secuencialidad controlada de los espacios académicos.
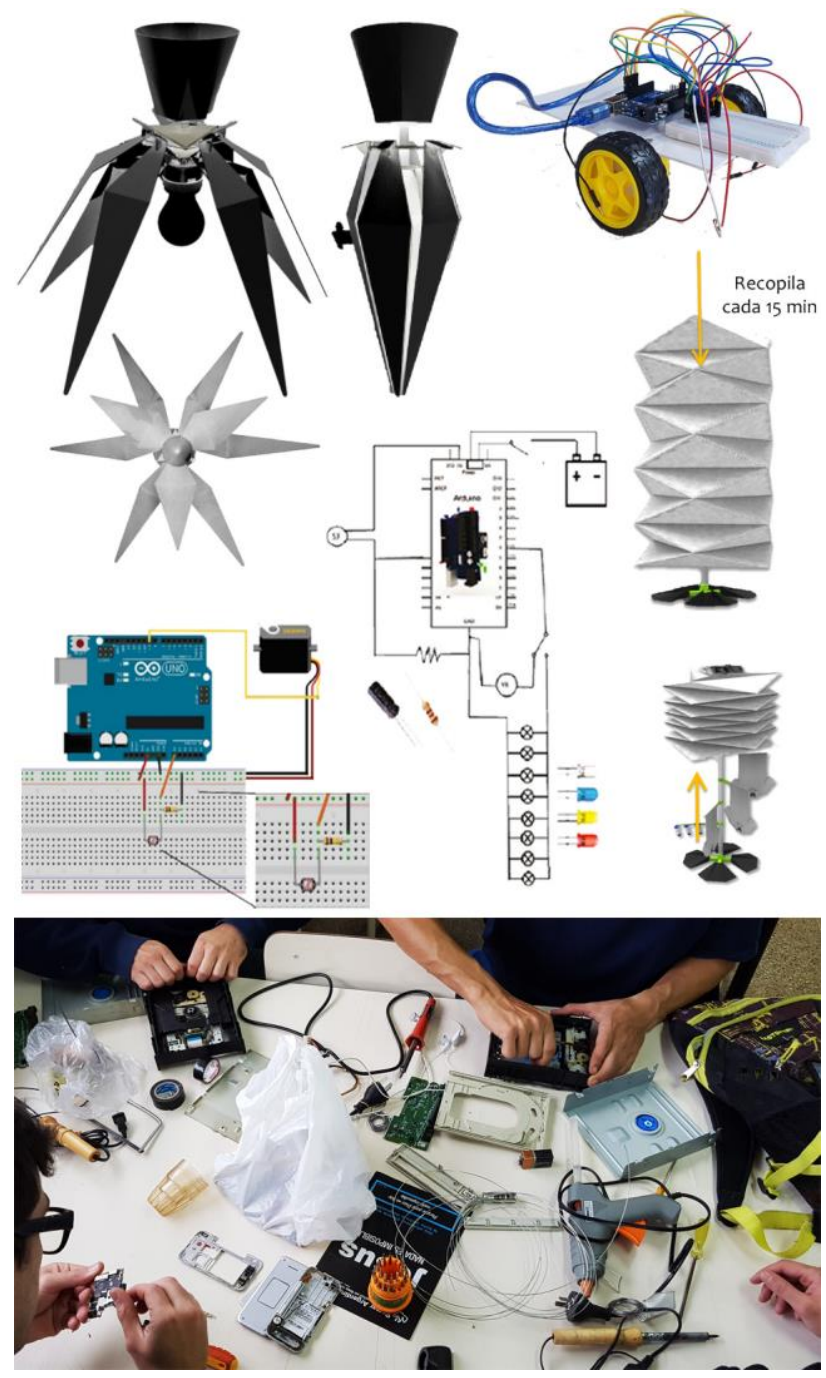

Figura 8: Dispositivos Reactivos (Microcontroladores y sensores). Grupos varios. Fuente: Autores.

Aprendizaje Colaborativo: La descentralización de la autoría desatada por Internet desafía bajo el slogan de Free Culture al modelo actual de propiedad intelectual dando nacimiento a la confrontación de fuertes argumentos culturales y desiguales condiciones económicas en el debate. Tecnologías digitales de distribución como las Redes P2P, propuestas de reformulación de los derechos de autor como Creative Commons; innovaciones del código abierto; Internet Zero; blogging, o movimientos como el del Software Libre, nos sitúan en un periodo de nuestra cultura que reclama una revisión del sistema legal dominante desde hace más de tres siglos. De esta manera aparece el CopyLeft como una aplicación de los principios del software libre al mundo del arte otorgando el derecho público a remezclar, modificar, desfigurar, trasformar, o simplemente copiar con solo mencionar al artista y la obra de origen. El público improvisa su arte y los artistas pueden reciclarse mutuamente con absoluta impunidad. Existe una inmensa cantidad de obra creativa en forma de base de datos, difundida por la red y de fácil acceso. Participar activamente de estos procesos fomenta el aprendizaje colaborativo.

\section{DISCUSION}

La correspondencia existente entre el diseño y las herramientas que han sido empleadas en su concepción nos remite a la discusión sobre la relación entre pensamiento y lenguaje. La relación consensuada entre ambos conceptos, nos habla de que cada diseño no es independiente de sus instrumentos proyectuales y lleva los signos y las marcas de los medios en la que ha sido pensado. El complejo mecanismo de percepción y de conocimiento entre el objeto y su imagen es condicionado y/o potenciado por los sistemas de representación y pre-figuración originando formas de lecturas y formas de memorias coherentes con los principios y leyes que los mismos determinan. (Chiarella, 1998)

Los ejercicios de cátedra presentados centran su problemática, no solo en el aprendizaje de los conocimientos y habilidades necesarias para manipular múltiples instrumentos de proyectación, sino en la comprensión de lo que estas herramientas de representación y prefiguración suponen para la concepción e ideación del diseño. La didáctica propuesta pretende orientar la formación del alumno hacia una actitud consciente y crítica del modo en el que los instrumentos y nuevas tecnologías utilizadas en la práctica disciplinar condicionan formas de interpretación, comprensión y acción de los fenómenos y problemas de nuestra realidad. Es así como abordamos el desafío de una actualización flexible y dinámica de nuestros procesos proyectuales. Para asumir el paradigma de la complejidad de lo digital en una didáctica proyectual contemporánea, es necesario tener criterios suficientes de lo que se conoce y los fundamentos de todas las opciones posibles para acompañar cualquier investigación y/o exploración con la mayor cantidad de recursos formales y técnicos posibles y en función de los objetivos del proyecto y del proceso iniciado. Es así como pretendemos adecuar los recursos tecnológicos disponibles en una visión integradora y estratégica para obtener una amplia capacidad en la construcción de los sistemas complejos que intervienen en el acto de la proyectación y el diseño.

El movimiento Maker amplía el campo de acción de las disciplinas proyectuales al incorporar nuevas herramientas y prácticas tecnopolíticas en la concepción, revisión y uso de artefactos tecnológicos que operan como bienes comunes, pudiendo contraponerse a los modelos dominantes de aplicación tecnológica. La creciente demanda de personalización de productos con la producción a medida y en pequeñas series sin aumento de costes de producción; la adaptabilidad contextual dinámica; exploración de sistemas reactivos de bajo costo; interfases mediante creación colectiva y diseño colaborativo proponen desplazamientos disciplinares desafíando a generar nuevos desarrollos competitivos que impactan tanto en la enseñanza y la didáctica del diseño como a nivel profesional.

\section{AGRADECIMIENTOS}

Becarios y Tesistas: Camila Picco, Nicolas Góngora. Pasantes en Investigación y Docencia: Nicolás Kakisu, Victoria Gaviola. Colaboradoras Dispositivos Reactivos: Taller de docsabotica.org (Mar delgado) - ideabotica.org 
en Rosa Futura (Curadoría: Carla Tortul) Plataforma de Arte + Ciencia +Tecnología en el MPBA Rosa Galisteo mayo 2017.

\section{REFERENCIAS}

Chiarella, M. (1998). La Imagen. Entre lo Analógico y lo Digital. POLIS, 1(4), 50-55. https://doi.org/10.14409/polis.v1i4.311

Chiarella M, Miotto Bruscato U, Castro Henriques G, Tortul C.B. (2018). Augmented Graphic Thinking. In: Marcos C. (eds) Graphic Imprints. EGA 2018. Springer, Cham. Berlin. https://doi.org/10.1007/978-3-319-93749-6_2

Dougherty, D, (2013). The maker mindset. Design, make, play: growing the next generation of stem innovators, 7-11.
González, L. F; Calvo, C. (2014) Desarrollo de competencias avanzadas en computación en la formación de los arquitectos latinoamericanos del siglo XXI In:. Proceedings of the 18th SIGraDi Conference, Montevideo, Uruguay. Universidad de la República, pp. 217-221.

Martini, S; Chiarella, M. (2017). Didactica Maker. Estrategias colaborativas de aprendizaje STEM en Diseño Industrial. p. 158-164 . Proceedings XX Congreso SIGraDi, Concepción. Chile

Stipech, A. (2012). Fundamentos de Cátedra Introducción a los Medios Digitales. FADU-UNL. Santa Fe. En: http://www. fadu.unl.edu.ar/imd (v.1-07-2018). 